\title{
A COVERING THEOREM FOR ODD TYPICALLY-REAL FUNCTIONS
}

\author{
E.P. MERKES \\ Department of Mathematical Sciences \\ University of Cincinnati \\ Cincinnati, Ohio 45221 U.S.A.
}

(Received July 20, 1979)

ABSTRACT. An analytic function $f(z)=z+a_{2} z^{2}+\ldots$ in $|z|<1$ is typically-real if $\operatorname{Im} f(z)$ Im $z \geq 0$. The largest domain $G$ in which each odd typically-real function is univalent (one-to-one) and the domain $\cap f(G)$ for all odd typically real functions $f$ are obtained.

KEY WORDS AND PHRASES. Typically-real functions, domain of univalence, covering theorems.

1980 MATHEMATICS SUBJECT CLASSIFICATION CODES. $30 \mathrm{C} 25$.

1. INTRODUCTION.

An analytic function $f(z)=z+a_{2} z^{2}+\ldots$ in the unit disk $E(|z|<1)$ is in the class $\mathrm{T}$ of typically-real functions if and only if there exists a nondecreasing function $\gamma$ on $[0, \pi]$ such that $\gamma(\pi)=1, \gamma(0)=0$, and

$$
f(z)=\int_{0}^{\pi} \frac{z d \gamma(t)}{1-2 z \cos t+z^{2}},
$$

$[1]$. The function $\gamma$ when normalized on $(0, \pi)$ by $\gamma(t)=(\gamma(t+)+\gamma(t-)) / 2$ is uniquely determined by $f$. 
The domain of univalence of the class $T$ is known [2] to be

$$
G=\{z:|z-1|<\sqrt{2}\} \cap\{z:|z+1|<\sqrt{2}\} .
$$

Brannon and Firwan [3] proved that the largest domain contained in $f(G)$ for every function in $\mathrm{T}$ is $|\mathrm{w}|<1 / 4$.

In this paper we obtain the corresponding results for the class $\mathrm{T}_{0}$ of odd typically-real functions. Recently Goodman [4] determined the largest domain that is contained in $f(E)$ for every $f \varepsilon T$. The analog of this result for the class $\mathrm{T}_{0}$ is an open problem.

2. The domain of univalence of $T_{0}$

THEOREM 2.1. The domain of univalence for $\mathrm{T}_{0}$ is the domain $\mathrm{G}$ of $(1.2)$. PROOF. Since $T_{0} \subset \mathrm{T}$, each $\mathrm{f} \in \mathrm{T}_{0}$ is univalent in $\mathrm{G}$. The theorem is estab1ished, therefore, if we can show that there is a function $f \varepsilon T_{0}$ that is not univalent in any domain $D$ that properly contains $G$. Let $f(z)=$ $z\left(1+z^{2}\right) /\left(1-z^{2}\right)^{2}=\frac{1}{2} z /(1-z)^{2}+\frac{1}{2} z /(1+z)^{2}$. This function is clearly in $T_{0}$ since $T$ is a linear class. The function

$$
\zeta=\frac{2 z}{1+z^{2}}
$$

maps $G$ onto $|\zeta|<1$. By the change of variables (2.1), the function $f$ has the form

$$
\begin{aligned}
f(z) & =\frac{1}{2} z /\left(1-2 z+z^{2}\right)+\frac{1}{2} z /\left(1+2 z+z^{2}\right) \\
& =\frac{1}{4} \zeta /(1-\zeta)+\frac{1}{4} \zeta /(1+\zeta)=\frac{1}{2} \zeta /\left(1-\zeta^{2}\right) .
\end{aligned}
$$

Since $\zeta /\left(1-\zeta^{2}\right)$ is not univalent in any domain that properly contains $|\zeta|<1$, we conclude that $f$ is not univalent in any domain that properly contains G.

3. A covering theorem for $T_{0}$

THEOREM 3.1. The largest domain $U$ contained in $f(G)$ for every $f \varepsilon T_{0}$ is the domain that includes the origin, is bounded in the right half-plane by $w=\rho e^{i \theta}$, 
where

$$
\rho=\left\{\begin{array}{ll}
(\cos \theta) / 2 & , 0 \leq|\theta| \leq \pi / 4, \\
1 /(4|\sin \theta|) & , \quad \pi / 4<|\theta| \leq \pi / 2,
\end{array}\right\}
$$

and is symmetric relative to the imaginary axis.

PROOF. By, (1.1) we have

$$
-f(-z)=\int_{0}^{\pi} \frac{z d \gamma(t)}{1+2 z \cos t+z^{2}}=\int_{0}^{\pi} \frac{z d[1-\gamma(\pi-\tau)]}{1-2 z \cos \tau+z^{2}} .
$$

If $f(z)=-f(-z)$, then by the uniqueness of $\gamma$ we have $\gamma(t)=1-\gamma(\pi-t)$ for $t \varepsilon[0, \pi]$. In particular, $\gamma(\pi / 2)=1 / 2$. For $f \varepsilon T_{0}$, therefore,

$$
\begin{aligned}
f(z) & =\int_{0}^{\pi / 2} \frac{z d \gamma(t)}{1-2 z \cos t+z^{2}}+\int_{\pi / 2}^{\pi} \frac{z d \gamma(t)}{1-2 z \cos t+z^{2}} \\
& =\int_{0}^{\pi / 2} \frac{z d \gamma(t)}{1-2 z \cos t+z^{2}}+\int_{\pi / 2}^{0} \frac{z d \gamma(\pi-t)}{1-2 z \cos (\pi-t)+z^{2}} \\
& =\int_{0}^{\pi / 2}\left[\frac{z}{1-2 z \cos t+z^{2}}+\frac{z}{1+2 z \cos t+z^{2}}\right] d \gamma(t)
\end{aligned}
$$

By the change of variables (2.1), we obtain

$$
f(z)=\int_{0}^{\pi / 2} \frac{\zeta d \gamma(t)}{1-\zeta^{2} \cos ^{2} t}, \gamma(\pi / 2)=1 / 2, \gamma(0)=0 .
$$

Let $z \varepsilon \partial G$. By (2.1) we have that the corresponding $\zeta$ is on the unit circle $|\zeta|=1$. For fixed $\zeta=e^{i \theta},-\pi<\theta \leq \pi$, the function $2 f(z)$ is by (3.1) in the closed convex hull $\mathrm{H}$ of the circular arc $w(s)=e^{i \theta} /\left(1-\mathrm{se}^{2 i \theta}\right), s \varepsilon[0,1]$. For each $\lambda, 0 \leq \lambda \leq 1$, the point $\lambda e^{i \theta}+(1-\lambda) i / \sin \theta$ is on the linear portion of $H$. Let $D(\lambda)$ denote the square of the distance from such a point to the origin. If $\theta \neq 0, \pi$, we have 


$$
D(\lambda)=\left|\lambda e^{i \theta}+\frac{1}{2}(1-\lambda) i / \sin \theta\right|^{2}=1+\left(1-\lambda^{2}\right) /\left(4 \sin ^{2} \theta\right) .
$$

This function of $\lambda$ has a minimum at $\lambda=1-2 \sin ^{2} \theta$ and $0 \leq \lambda \leq 1$ provided $|\sin \theta| \leq \sqrt{2} / 2$. Thus, the distance from any point of $H$ to the origin is not less than $\left[D\left(1-\sin ^{2} \theta\right)\right]^{\frac{1}{2}}=|\cos \theta|$ when $0<|\theta| \leq \pi / 4$ or $3 \pi / 4 \leq|\theta|<\pi$. For other $\theta \varepsilon(-\pi, \pi]$ the distance from any point of $H$ to the origin is $\min \{1,1 /(2|\sin \theta|)\}=1 /(2|\sin \theta|)$ for $\pi / 4<|\theta|<3 \pi / 4$ and 1 for $\theta=0$ or $\pi$. Since the convex hull $\mathrm{H}$ contains for each $z \in \partial G$ the values of $2 f(z)$ for all $f \varepsilon T_{0}$ and since every point of $H$ is the value of $2 f(z)$ for some $f \varepsilon T_{0}$, we conclude that $U$ is the exact domain covered by all $f \in T_{0}$ when $z \in G$.

\section{REFERENCES}

1. Robertson, M. S., On the coefficients of a typically-real function, Bull. Amer. Math. Soc., 41 (1935), 565-572.

2. Merkes, E. P., On typically-real functions in a cut plane, Proc. Amer. Math. Soc., 10 (1959), 863-868.

3. Brannan, D. A. and Kirwan, W. E., A covering theorem for typically-real functions, Glasgow Math. J., 10 (1969), 153-155.

4. Goodman, A. W., The domain covered by a typically-real function, Proc. Amer. Math. Soc., 64 (1977), 233-237. 


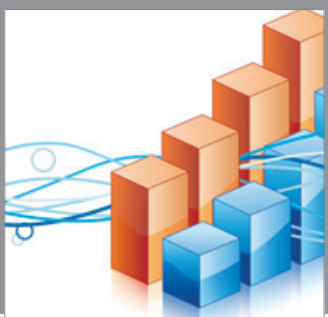

Advances in

Operations Research

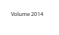

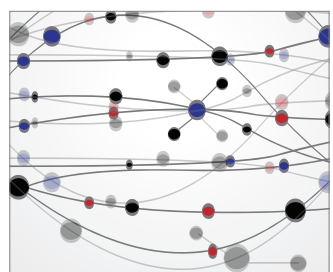

\section{The Scientific} World Journal
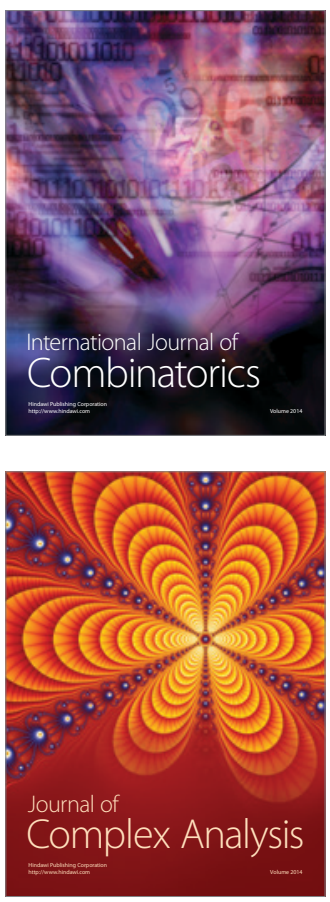

International Journal of

Mathematics and

Mathematical

Sciences
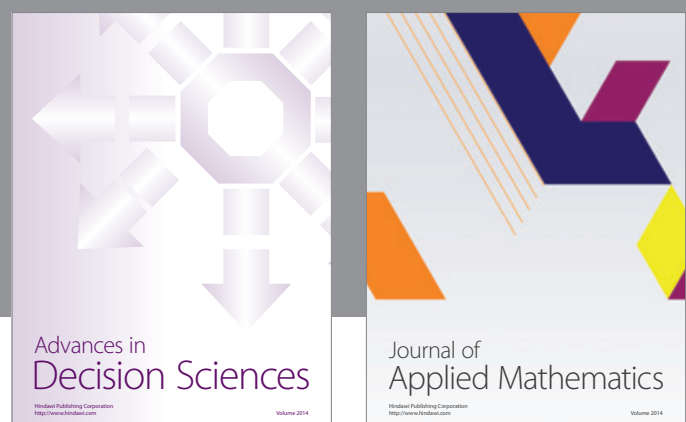

Journal of

Applied Mathematics
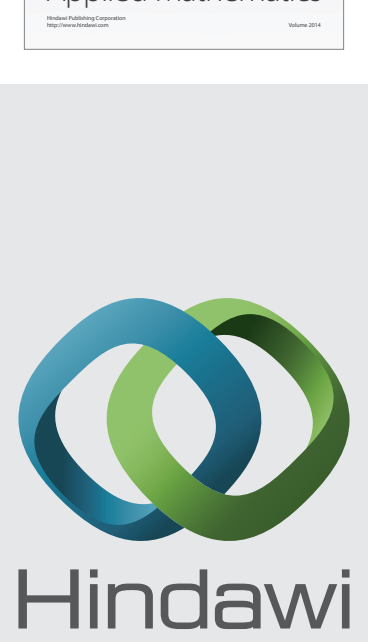

Submit your manuscripts at http://www.hindawi.com
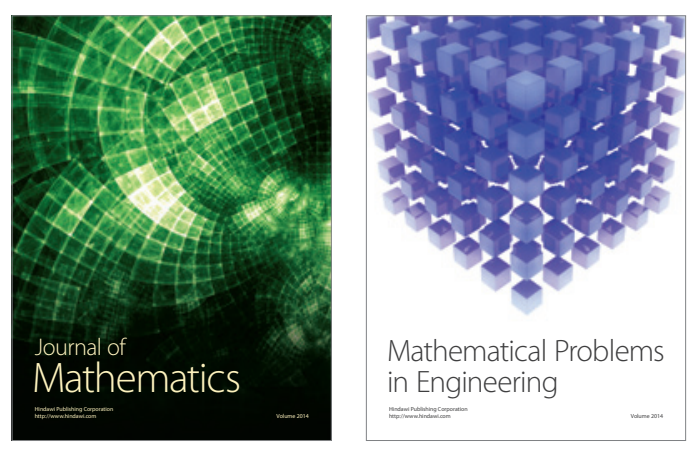

Mathematical Problems in Engineering
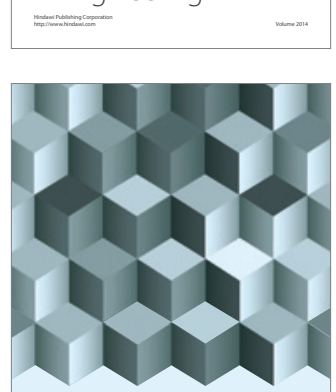

Journal of

Function Spaces
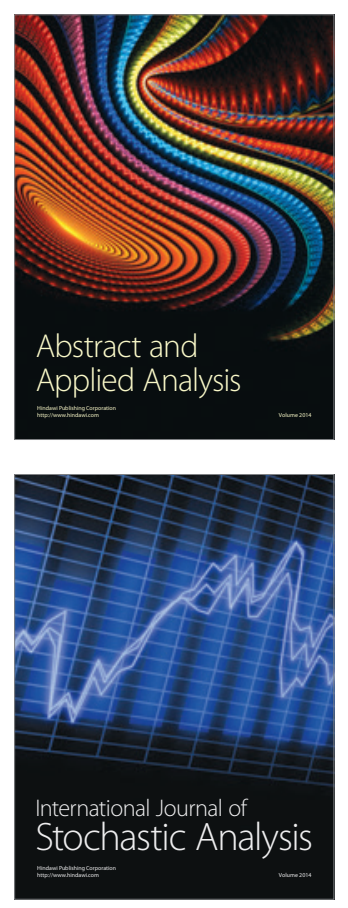

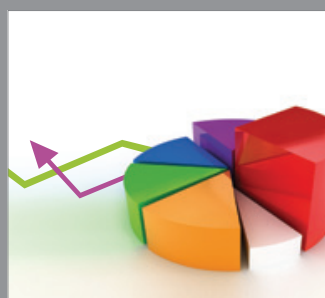

ournal of

Probability and Statistics

Promensencen
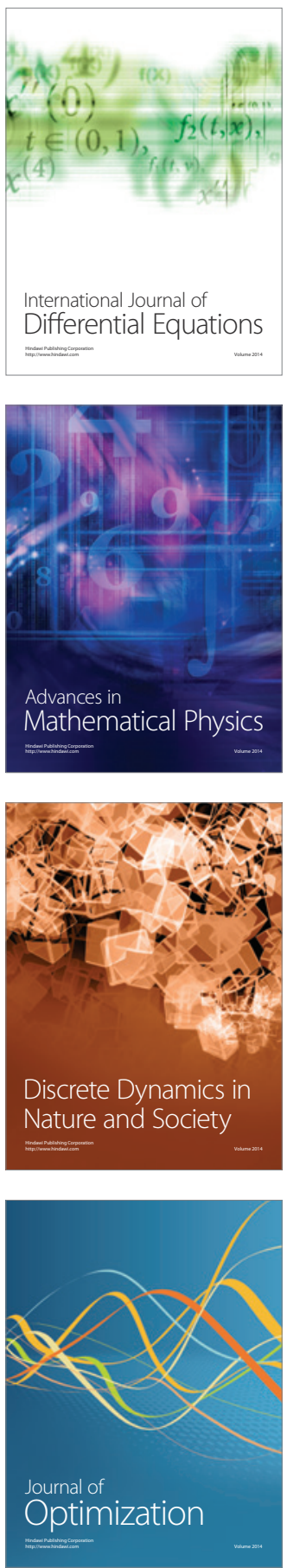